\title{
Assistência multidisciplinar no acompanhamento de pacientes com pênfigo vulgar
}

Multidisciplinary assistance in the follow-up of patients with pemphigus vulgar

Asistencia multidisciplinar en el seguimiento de pacientes con pulfia vulgar

Weslay Rodrigues DA SILVA

Residente em Odontologia Hospitalar com ênfase em Terapia Intensiva COREMU UFPE, Real Hospital Português de Beneficência,

52010-075 Recife - PE, Brasil

.https://orcid.org/0000-0002-0430-4656

Hannah Gil de Farias MORAIS

Doutoranda, Programa de Pós-Graduação em Ciências Odontológicas, Universidade Federal do Rio Grande do Norte (UFRN) 59056-000 Natal - RN, Brasil

https://orcid.org/0000-0003-3488-3465

Lucas Melo DA COSTA

Mestrando, Programa de Pós-Graduação em Ciências Odontológicas, Universidade Federal do Rio Grande do Norte (UFRN)

59056-000 Natal - RN, Brasil https://orcid.org/0000-0003-3321-3689 Thyago Morais Vicente DA SILVA

Doutorando, Programa de Pós-Graduação em Odontologia, Universidade Federal de Pernambuco (UFPE), 50670-901 Recife - PE, Brasil https://orcid.org/0000-0002-1841-6560 Thayanara Silva MELO

Doutoranda, Programa de Pós-Graduação em Odontologia, Universidade Federal de Pernambuco (UFPE), 50670-901 Recife - PE, Brasil https://orcid.org/0000-0002-4184-4635

\section{Resumo}

Este trabalho relata o caso clínico de uma paciente do sexo feminino, 33 anos, com queixa principal de múltiplas ulcerações em mucosa bucal, a qual havia sido previamente diagnosticada com pênfigo vulgar, porém devido aos efeitos adversos da terapia medicamentosa abandonou o tratamento. Foi objetivada a retomada da adesão da paciente ao tratamento, com auxílio multiprofissional, tornando-a corresponsável pela sua melhora clínica e a terapêutica envolveu o uso tópico de pomada de Propionato de Clobetasol $0,05 \%$ e a administração sistêmica de Prednisona, atingindo resultados satisfatórios pela ótima evolução clínica.

Descritores: Pênfigo; Dermatopatias Vesiculobolhosas; Doenças Autoimunes.

\section{Abstract}

The aim of this paper is report a case of a 33-year-old female patient, with the main complaint of multiple ulcerations in the oral mucosa, who had previously been diagnosed with pemphigus vulgaris, but due to the adverse effects of drug therapy, she abandoned the treatment. It was intent to the patient's adherence to treatment, with multiprofessional assistance, making her co-responsible for her clinical improvement. The therapy involved the topical use of $0.05 \%$ Clobetasol Propionate ointment and the systemic administration of Prednisone, achieving satisfactory results by the excellent clinical evolution.

Descriptors: Pemphigus; Skin Diseases, Vesiculobullous; Autoimmune Diseases.

\section{Resumen}

En este trabajo se aborda el caso clínico de una paciente de 33 años, con el síntoma principal de múltiples úlceras en la mucosa oral, que previamente había sido diagnosticada de pénfigo vulgar, pero que por los efectos adversos de la farmacoterapia abandonó el tratamiento. . El objetivo fue retomar la adherencia de la paciente al tratamiento, con asistencia multiprofesional, haciéndola corresponsable de su mejoría clínica y la terapia involucró el uso tópico de pomada de Propionato de Clobetasol al $0.05 \%$ y la administración sistémica de Prednisona, logrando resultados satisfactorios. por la excelente evolución clínica.

Descriptores: Pénfigo; Enfermedades Cutáneas Vesiculoampollosas; Enfermedades Autoinmunes.

\section{INTRODUÇÃO}

O pênfigo corresponde a um grupo de doenças dermatológicas, autoimune, de caráter crônico, representada clinicamente por lesões vesículo-bolhosas e acometimento mucocutâneo. Genericamente é classificada em diversos subtipos, tais como: vulgar, foliáceo, induzido por drogas, paraneoplásico $\mathrm{e}$ associado a $\lg \mathrm{A}^{1,2}$. O perfil epidemiológico envolve pacientes entre a quarta e sexta décadas de vida, com leve predileção pelo sexo feminino.

O pênfigo vulgar é o mais comumente observado em mucosa oral e possui incidência aproximada na população geral de 0,1 a 0,5 casos a cada 100.000 indivíduos por ano ${ }^{3,4}$. As lesões observadas em cavidade oral geralmente encontram-se ulceradas, pois, devido à fragilidade das bolhas formadas, são facilmente rompidas, deixando uma superfície dolorida, eritematosa, irregular, podendo ser recobertas por pseudomembrana. As lesões orais costumam anteceder lesões cutâneas e de outras mucosas. Embora sejam as primeiras a aparecer, podem ser mais resistentes ao tratamento, e de difícil remissão ${ }^{1}$.

O tratamento desses pacientes é realizado preferencialmente com 0 uso de corticosteróides sistêmicos, em que são administradas doses gradativamente menores até a remissão dos sintomas, bem como o estabelecimento uma dose mínima de manutenção. Drogas imunorreguladoras e corticosteróides tópicos (como o Propionato de Clobetasol) também podem ser utilizados na terapêutica. Além disso, é de fundamental importância 0 tratamento multidisciplinar, envolvendo médico, estomatologista e psicológico, devido à complexidade da doença e efeitos adversos da terapia, bem como retornos periódicos para avaliação da evolução dos pacientes ${ }^{5}$.

O objetivo deste trabalho é relatar o caso 
de uma paciente diagnosticada previamente com pênfigo vulgar, que suspendeu a terapia medicamentosa por conta própria e evoluiu com múltiplas ulcerações em mucosa bucal, evidenciando a importância do tratamento multidisciplinar desses pacientes.

CASO CLÍNICO

Paciente do sexo feminino, 33 anos, compareceu ao departamento de medicina oral com queixa principal de ulcerações múltiplas na cavidade oral presentes nos últimos 2 meses. Durante a anamnese, informou ter sido diagnosticada com pênfigo vulgar (Figura 1) há cerca de 3 anos, realizava tratamento com dose mínima de corticoestóide, mas que resolveu suspender o tratamento devido ao ganho de peso nos últimos meses.

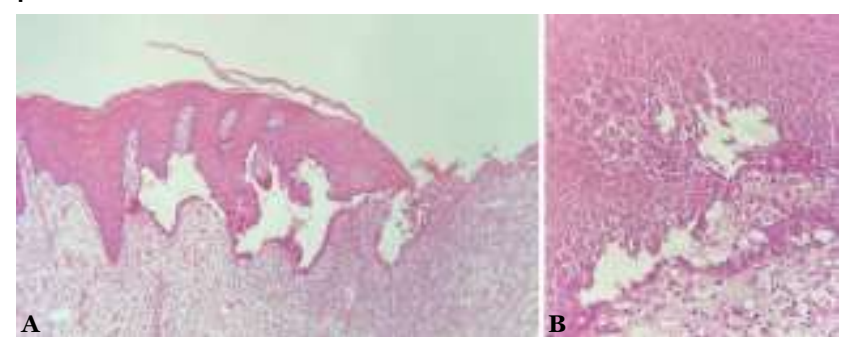

Figura 1: Achados histopatológicos. A) Fenda intraepitelial, observada no terço inferior do epitélio (HE, 100x). B) Evidencia-se, no epitélio, espongiose, exocitose e acantólise suprabasal (HE, 400x).

Ao exame extraoral, não se observou presença de lesões cutâneas, bem como nenhum outro sinal relevante. No exame intraoral, foram observadas ulcerações difusas com superfície coberta por membrana fibrinopurulenta circundadas por um halo eritematoso na região posterior de mucosa jugal direita e palato mole (Figura $2 \mathrm{~A}$ e $\mathrm{B}$ ), foi observado a presença de "gengivite descamativa" (Figura 2C) envolvendo toda a gengiva inserida inferior. A paciente relatou dor intensa nas regiões afetadas e grande dificuldade para se alimentar.

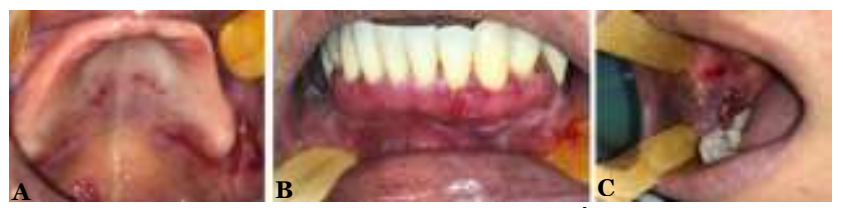

Figura 2. Característica clínica inicial. A) Úlcera em região de palato duro. B) Gengivite descamativa em gengiva inferior. C) Úlcera em mucosa jugal.

O tratamento local consistiu no uso tópico de pomada de Propionato de Clobetasol 0,05\% aplicado nas regiões de úlcera $3 x$ por dia, nas lesões gengivais foi aplicado diariamente por meio de uma moldeira acrílica individual nas áreas correspondentes a toda a gengiva inserida inferior. $O$ tratamento sistêmico foi retomado, a administração de prednisona (30 $\mathrm{mg} /$ dia por 15 dia) e a diminuição da dose foi realizada a cada 15 dias, até chegar à dose mínima novamente (5 mg/dia). Após duas semanas do início do tratamento, a paciente já apresentava boa evolução clínica (Figura 3). A paciente também foi encaminhada a psicoterapia para prevenção de possíveis fugas do tratamento.

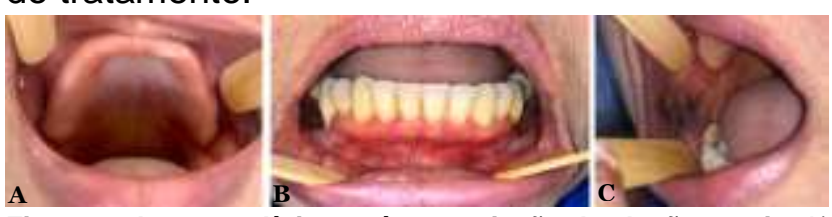

Figura 3. Aspecto clínico após a remissão das lesões orais. A) Palato duro. B) Gengiva inferior. C) Mucosa jugal.

DISCUSSÃO

O pênfigo apesar de sua baixa ocorrência, por muitos anos esteve associada a índices elevados de mortalidade, os quais foram radicalmente reduzidos desde a introdução da terapia medicamentosa ${ }^{6}$. Apresenta cinco variantes: pênfigo vulgar, foliáceo, paraneoplásico, induzido por drogas e pênfigo associado a $\lg \mathrm{A}^{7}$. No entanto, lesões orais estão restritas ao vulgar e paraneoplásico ${ }^{8}$.

O pênfigo vulgar é uma condição mucocutânea, sistêmica e vesículo-bolhosa ${ }^{9}$. O surgimento de lesões mucosas e cutâneas pode ser explicado a partir da fisiopatologia da doença, que se desenvolve a partir da produção de autoanticorpos contra proteínas essenciais à integridade e união do tecido epitelial - as desmogleínas 1 e 3 (DSG-1 e DSG-3). Essas proteínas são constituintes dos desmossomos e pertencem a uma família de caderinas que agem como moléculas de adesão celular. Logo, uma resposta imune às desmogleínas leva à perda de adesão dos queratinócitos, levando à acantólise e à formação de fendas intraepiteliais ${ }^{2,10,11}$.

O pênfigo vulgar é o tipo predominante, apresentando incidência variando de 0,5 a 3,2 casos em cada 100.000 indivíduos/ano ${ }^{12}$. Embora epidemiologicamente não haja de predileção por sexo, alguns estudos relataram uma leve prevalência por mulheres, com proporção de 2:1, e incidência entre a $4^{a}$ e $6^{a}$ décadas de vida. Alguns casos raros também foram relatados em crianças e idosos ${ }^{13}$. A paciente do caso em questão apresenta o pênfigo vulgar, cujo sexo e idade estão em consonância com a literatura.

A mucosa oral é o local inicial de envolvimento em $70 \%$ a $90 \%$ dos casos antes do envolvimento da pele e de outras mucosas, e em alguns casos, pode ser o único local afetado $^{8}$. Embora não haja predileção por área, as lesões podem estar localizadas em qualquer região sujeita a trauma por atrito, como mucosa 
jugal, palato mole, lábio inferior e língua e, com menor frequência, na gengiva, sendo a gengivite descamativa a manifestação mais comum da doença quando há envolvimento gengival $^{13}$. Após interrupção terapêutica, a paciente apresentava úlceras restritas a mucosa oral, em mucosa jugal, palato e gengiva.

As lesões orais podem variar de úlceras bastante superficiais a pequenas vesículas ou bolhas que se rompem rapidamente, deixando uma área erosiva com um halo eritematoso. As lesões cutâneas, quando presentes, geralmente são subsequentes às manifestações orais, podendo surgir como simples erupções cutâneas a erosões, vesículas, bolhas ou úlceras $^{12,14}$.

A maioria das terapias visa melhorar os sintomas através da redução de autoanticorpos séricos, diretamente ou através da supressão imunológica, melhorando a qualidade de vida. $\mathrm{Na}$ maioria dos pacientes, isso se traduz clinicamente na ausência ou pela formação de bolhas ocasionais ${ }^{10,15}$. Quando existem apenas lesões orais, os corticosteróides são aplicados topicamente em formulações de enxágue (solução) ou pomada. Se houver lesões cutâneas, 0 tratamento consiste em corticoesteróides sistêmicos que pode ser combinado com medicamentos imunomoduladores ou imunossupressores adjuvantes $^{9,10,15,16}$. Foi prescrito corticoesteróide tópico para tratamento das lesões orais e sistêmico, já que a paciente apresentava histórico de lesões cutâneas, embora no momento da consulta as manifestações fossem restritas a mucosa oral.

O pênfigo está associado a relativa morbimortalidade e tem impacto psicossocial significativo, refletindo negativamente na qualidade de vida dos pacientes. Vários fatores estão relacionados, dentre os quais, os mais importantes são a gravidade clínica da doença e a dose terapêutica de corticoesteróide, devido aos seus efeitos adversos, como, infecções, ganho de peso, insuficiência adrenal e osteoporose $e^{15,17}$.

O pênfigo vulgar tem curso prolongado, com períodos de remissão e recidiva, que associado aos efeitos colaterais da terapia podem causar danos psicológicos. Cerca de $70 \%$ dos pacientes com PV estão insatisfeitos com sua aparência ${ }^{18}$. Esses pacientes devem ser acompanhados por uma equipe multidisciplinar, composta por oftalmologista, dermatologista, otorrinolaringologista, cirurgiãodentista, imunologista e psicólogo, devido à complexidade dos fatores inerentes à condição $^{19}$. A paciente estudada é acompanhada por equipe multidisciplinar, embora estivesse apresentando boa resposta a terapia decidiu abandonar o tratamento por consequência ao ganho de peso. Devido a presença das lesões orais retornou ao nosso serviço, que além do tratamento farmacológico, foi encaminhada a psicoterapia a fim de evitar novo abandono do tratamento.

CONCLUSÃO

O tratamento multidisciplinar com abordagem integrada deve ser empregado sempre que possível para os pacientes com pênfigo vulgar, devido à complexidade da dermatose e dos efeitos colaterais do tratamento, proporcionando um suporte resolutivo e contínuo, de forma a minimizar o impacto e aumentar a adesão à terapia proposta, melhorando a qualidade de vida do paciente.

REFERÊNCIAS

1. Griffiths C, Barker J, Bleiker T, Chalmers R, Creamer D. Rook's textbook of dermatology. 9. ed. Londres: Wiley Blackwell; 2016.

2. Feller L, Ballyram R, Khammissa RA, Altini M, Lemmer J. Immunopathogenic oral diseases: an overview focusing on pemphigus vulgaris and mucous membrane pemphigoid. Oral Health Prev Dent. 2017; 15(2):177-82.

3. Baum S, Sakka N, Artsi O, Trau H, Barzilai A. Diagnosis and classification of autoimmune blistering diseases. Autoimmun. Rev. 2014; 13(4-5):482-89.

4. Mcmillan R, Taylor J, Shephard M, Ahmed $R$, Carrozzo M, Setterfield J, et al. World Workshop on Oral Medicine VI: a systematic review of the treatment of mucocutaneous pemphigus vulgaris. Oral Surg Oral Med Oral Pathol Oral Radiol Endod. 2015;120(2):132-42.

5. Ferreira VYN, Lyra TC, Pereira LL, Nascimento MAB, Souza LB, Bonan PRF. Eficácia do uso de corticosteroide sistêmico no tratamento do pênfigo vulgar oral. Arq ciênc saúde. 2016; 23(3):10-13.

6. Madala J, Bashamalla R, Kumar MP. Conceitos atuais de pênfigo com uma visão profunda de seus aspectos moleculares. J Oral Maxillofac Pathol. 2017;21:260-63.

7. Ormond $M, M c P a r l a n d \quad H$, Donaldson ANA, Andiappan M, Cook RJ, Escudier M et al. An Oral Disease Severity Score validated for use in oral pemphigus vulgaris. $\mathrm{Br} J$ Dermatol. 2018;179(4):872-81.

8. Kumar SJ, Nehru Anand SP, Gunasekaran N, Krishnan R. Oral pemphigus vulgaris: A case report with direct immunofluorescence study. J Oral Maxillofac Pathol. 2016;20(3):549.

9. Dimarco C. Pemphigus: Pathogenesis to Treatment. R. I. Med. J. 2016;99(12):28-31. 
10. Santoro FA, Stoopler ET, Werth VP. Pemphigus. Dent Clin North Am. 2013;57(4): 1-20.

11. Batista MIBH, Paulino MR, Santos CAO, Andrade SC, Lima CA, Gueiros LA, et al. Correlação entre anti-desmogleína e lesões mucocutâneas em pacientes com pênfigo vulgar ou foliáceo. REFACS. 2019;7(1):16-22.

12. Saccucci M, Di Carlo $G$, Bossù $M$, Giovarruscio F, Salucci A, Polimeni A. Autoimmune Diseases and Their Manifestations on Oral Cavity: Diagnosis and Clinical Management. J. Immunol. Res. 2018; 2018:6061825.

13. Arpita R, Monica A, Venkatesh N, Atul S, Varun M. Oral Pemphigus Vulgaris: Case Report. Ethiop. J. Health Sci. 2015;25(4):367-72.

14. Magliocca KR, Fitzpatrick SG. Autoimmune Disease Manifestations in the Oral Cavity. Surg. Pathol. Clin. 2016;10(1):57-88.

15. Kasperkiewicz M, Ellebrecht CT, Takahashi H, Yamagami J, Zillikens D, Payne AS, et al. Pemphigus. Nat Rev Dis Primers. 2017; 11(3):17026.

16. Sankar V, Noujeim M. Oral Manifestations of Autoimmune and Connective Tissue Disorders. Atlas Oral Maxillofac Surg Clin North Am. 2017;25(2):113-26.

17.Sung JY, Roh MR, Kim SC. Quality of Life Assessment in Korean Patients With Pemphigus. Ann Dermatol. 2015;27(5):492-98.

18. Rao R, Srinivas CR. Counseling in pemphigus. Indian J Dermatol. 2017;62:279-81.

19. Hansen MS, Klefter ON, Julian HO, Pedersen AML, Heegaard S. Management of patients with ocular manifestations in vesiculobullous disorders affecting the mouth. Oral Dis. 2017; 23(7):849-53.

\section{CONFLITO DE INTERESSES}

Os autores declaram não haver conflitos de interesse

AUTOR PARA CORRESPONDÊNCIA

\section{Thayanara Silva Melo}

Rua Osvaldo Guimarães, ํㅡ 30

Recife-PE CEP: $506.70-330$

Contato: +55 (81) 9.9874-6438

E-mail: thayanaramelo@hotmail.com

Submetido em 02/12/2020

Aceito em 18/07/2021 